\title{
Pubarquia. Adrenarquia. Hirsutismo
}

B. García Cuartero

Servicio de Pediatría. Endocrinología Pediátrica. Hospital Severo Ochoa. Leganés, Madrid. España.

Rev Pediatr Aten Primaria. 2009; / / Supl I6:s / 43-s /54

Beatriz García Cuartero, bgarciac.hsvo@salud.madrid.org

\section{Resumen}

La pubarquia precoz es motivo frecuente de consulta en Endocrinología pediátrica. Es más frecuente en niñas y su presencia no implica necesariamente el inicio de la pubertad. La adrenarquia es la causa más frecuente de pubarquia precoz y se debe al aumento de la producción de andrógenos por la maduración fisiológica de la glándula suprarrenal. Exige una valoración clínica adecuada para descartar patología, como la hiperplasia suprarrenal forma tardía. Estudios recientes demuestran que las niñas con pubarquia prematura, en particular con antecedente de bajo peso al nacer, tienen mayor riesgo de presentar, en la etapa puberal y postpuberal, hirsutismo y alteraciones menstruales, manifestaciones clínicas del síndrome de ovario poliquístico (SOP) debido al hiperandrogenismo. En estos casos el tratamiento se basa en la utilización de anticonceptivos durante al menos 6 meses, añadiendo un antiandrógeno si la respuesta es escasa, y el empleo de técnicas de cosmética dependiendo de cada paciente.

Palabras clave: Pubarquia precoz, Adrenarquia, Hirsutismo, Hiperandrogenismo, Síndrome de ovario poliquístico.

\section{Abstract}

Premature pubarche is one of the chief complaints in paediatric endocrinology. It appears to be more common in girls, and seems to be unrelated to the pubertal maturation.

Adrenarche is the most common cause of premature pubarche due to a maturational increase in adrenal androgen production. A carefully exam should be performed to exclude other causes of premature pubarche as non classic congenital adrenal hyperplasia. Recently, longitudinal studies have showed that girls with premature pubarche, particulary those small for gestational age, went on to develop oligomenorrhea, and hirsutism, during puberty, all criteria for polycystic ovary syndrome (PCOS). Treatment for these cases include oral contraceptives, adding antiandrogens if the response is suboptimal after 6 months, and hair removal therapy.

Key words: Premature pubarche, Adrenarche, Hirsutism, Hyperandrogenism, Polycysticovary syndrome.

La autora declara no presentar conflictos de intereses en relación con la preparación y publicación de este artículo. 
Pubarquia precoz; adrenarquia precoz Se denomina pubarquia precoz a la presencia de vello grueso y rizado en el pubis sin otros caracteres sexuales secundarios, en las niñas antes de los 8 años y en los niños antes de los 9 años de edad. $A$ veces se acompaña también de axilarquia, acné, aumento del olor corporal y piel grasa. En muchos casos no se asocia a ninguna patología de base y entonces hablamos de adrenarquia precoz.

\section{Etiopatogenia}

Esta alteración se produce 2 o más años antes de iniciarse la secreción nor- mal de gonadotropinas y esteroides sexuales, y se debe al aumento de la síntesis de varios andrógenos adrenales en la zona reticular (sulfato-dehidroepiandrosterona (DHEAS) y androstenodiona) por aumento de la actividad de las enzimas $17-\mathrm{OH}-\alpha$-hidroxilasa y 17,20 liasa, presumiblemente debido a la maduración precoz de la glándula suprarrenal (figura 1).

Aunque los mecanismos exactos de este fenómeno no han sido del todo aclarados, recientemente algunos autores han implicado a la angiotensina II como responsable, al favorecer la proli-

Figura 1. Esquema de las diferentes vías enzimáticas que participan en la biosíntesis de hormonas esteroideas en la glándula suprarrenal.

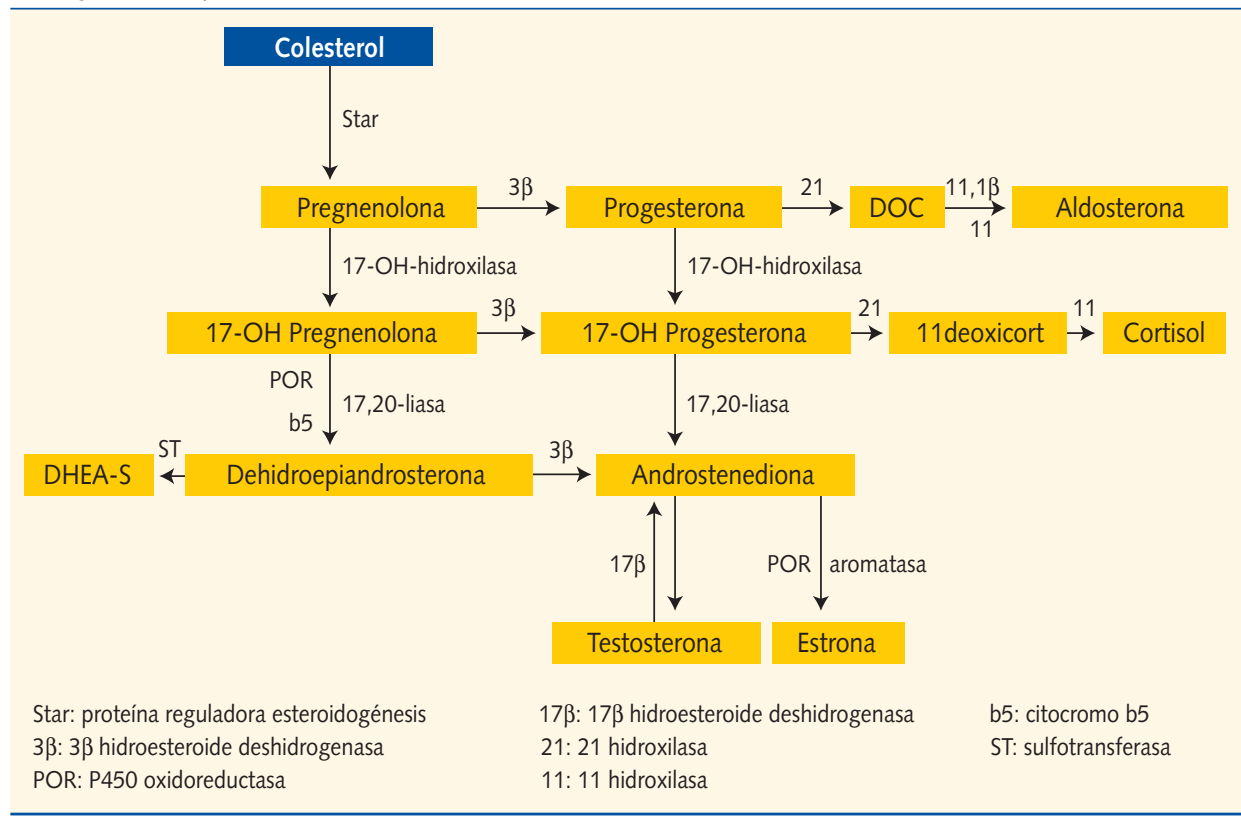


feración celular y la esteroidogénesis a través de receptores tipo 1 y 2 (AT1, AT2). Sin embargo, no se ha encontrado hasta el momento ninguna alteración en los genes que codifican dichos receptores ${ }^{1}$. En los últimos años se ha relacionado también la presencia de pubarquia precoz con polimorfismos del gen del receptor del IGF-I y del receptor androgénico, explicando la mayor sensibilidad de los folículos pilosos a las hormonas esteroideas ${ }^{2-4}$.

Desde el punto de vista epidemiológico se desconoce exactamente su prevalencia en la población general. Es más frecuente en mujeres que en hombres, siendo motivo de consulta muy frecuente en endocrinología pediátrica.

Aunque la causa más frecuente es la adrenarquia precoz, su presencia obliga a descartar patologías en las que la pubarquia puede ser la primera manifestación clínica, sobre todo, la hiperplasia suprarrenal congénita forma tardía (87\% presentan pubarquia precoz), la pubertad precoz, o tumores virilizantes.

Por otro lado, la presencia de pubarquia no se debe confundir con el inicio de la pubertad, ya que esta se manifiesta en las niñas con la aparición del botón mamario y en los niños con el aumento del volumen testicular ( $\geqslant 4 \mathrm{ml}$ de Prader).

\section{Enfoque diagnóstico}

\section{y pruebas complementarias}

Además de los datos clínicos reseñados, el aumento de la producción de andrógenos adrenales produce aumento de la velocidad de crecimiento y aceleración moderada de la edad ósea.

El vello púbico una vez aparecido sigue evolucionando de forma progresiva, completando su desarrollo. Tras el vello púbico puede aparecer la axilarquia, incluso en algunos casos es a la inversa.

Los niños/as que presentan pubarquia precoz aislada suelen ser más altos que sus compañeros al inicio de la pubertad, pero inician la pubertad a la misma edad y alcanzan una talla final igual.

La obesidad favorece esta condición 5 . Además es importante reseñar que en los últimos años, L. Ibáñez y cols. demostraron en población catalana que las niñas con pubarquia precoz que presentaban bajo peso al nacer con un incremento rápido postnatal del mismo, tienen un riesgo elevado de hiperandrogenismo ovárico funcional y con ello de resistencia insulínica, diabetes mellitus tipo 2 y síndrome metabólico. Esta secuencia es más probable cuanto menor peso al nacer, ${ }^{6,7}$. Sin embargo, estos datos no han sido corroborados en otras poblaciones estudiadas $^{8}$. En los casos de adrenarquia exage- 


\begin{tabular}{ll}
\hline Tabla I. Causas postnatales de hiperandrogenismo \\
\hline Origen suprarrenal & Pubarquia simple \\
& Hiperandrogenismo suprarrenal funcional \\
& (síndrome de ovarios poliquísticos) \\
& Tumores \\
& Síndrome de Cushing \\
& Hiperplasia suprarrenal congénita \\
& Resistencia a los glucocorticoides \\
\hline Origen ovárico & Hiperandrogenismo ovárico funcional \\
& (síndrome de ovarios poliquísticos) \\
& Tumores virilizantes \\
\hline Origen testicular & Tumores \\
& Testotoxicosis \\
\hline Aumento de la producción periférica & Obesidad; hiperprolactinemia \\
& Hiperandrogenismo idiopático \\
\hline Exógeno: administración de fármacos & Hidantoínas, corticoides, esteroides, ciclosporina, diazóxido, etc. \\
\hline
\end{tabular}

rada los niveles de DHEA-s y androstenodiona muy elevados favorecerían dicha secuencia (tabla I), y la pubarquia precoz sería expresión de hiperandrogenismo en la etapa prepuberal, debido presumiblemente a una disfunción congénita de la esteroidogénesis ${ }^{9,10}$.

Ante la presencia de un/a paciente con pubarquia precoz hay que preguntar: ¿desde cuándo presenta dicha pubarquia?, ¿se acompaña de otros caracteres sexuales secundarios?, ¿ha habido un aumento de la velocidad de crecimiento y del olor corporal o un aumento importante del peso o presencia de acné?

Es importante conocer el desarrollo de la pubertad en los padres y familiares, así como la existencia de familiares con problemas con el vello, de fertilidad, trastor- nos menstruales, diabetes mellitus tipo 2 , hiperandrogenismo e hiperplasia suprarrenal.

En los antecedentes personales investigar la existencia de bajo peso al nacer y la ingesta de medicación (corticoides...), así como la evolución del peso y la talla.

En la exploración recoger el peso, la talla, el índice de masa corporal (IMC) y valorar la velocidad de crecimiento, la presencia o no de otros caracteres sexuales secundarios, acné, acantosis nígricans y datos de virilización como hipertrofia de clítoris, hirsutismo, aumento de la musculatura y atrofia mamaria.

Con estos datos es posible hacer un enfoque diagnóstico de aproximación, que en la mayoría de los casos lleva al 
diagnóstico de sospecha de adrenarquia precoz.

Si existen dudas y los datos clínicos lo avalan, pedir una edad ósea, y si está adelantada respecto a la edad cronológica obliga al despistaje de patología, fundamentalmente la hiperplasia suprarrenal congénita en su forma de presentación tardía; realizar un test de ACTH para 17$\mathrm{OH}$-progesterona, ya que la determinación basal de estos andrógenos no lo descarta, sobre todo si la velocidad de crecimiento y la edad ósea están aceleradas. Si se confirma la sospecha, solicitar el estudio genético e iniciar tratamiento con corticoides.

Si presentan signos de importante virilización es necesario descartar otras patologías, como la tumoral.

Si no presenta caracteres sexuales secundarios ni virilización y la edad ósea es acorde con la edad cronológi$\mathrm{ca}$, se trataría de una adrenarquia pre$\mathrm{coz}$, por lo que la paciente no precisaría tratamiento específico, salvo evitar el sobrepeso, y, por tanto, la actitud clínica será de observación por las posibles alteraciones tras la pubertad, sobre todo en las niñas con antecedentes reseñados, ya que hay un $45 \%$ que aproximadamente 3 años después de la menarquia pueden presentar hiperandrogenismo ovárico funcional, hiperinsulinemia e hiperlipemia (sobre todo las que tuvieron bajo peso al nacer, aunque es aún motivo de controversia ${ }^{11}$ ).

\section{Hirsutismo}

Crecimiento de vello en zonas andrógeno-dependientes que no existe en condiciones normales: región supralabial, patillas, barbilla, línea anterior del tórax, areolas mamarias, línea alba, cara interna de muslos e ingles y espalda. Este vello tiende a ser más grueso y oscuro y se puede asociar a acné, caída del cabello, e irregularidades menstruales. Es importante diferenciar, y a veces no es fácil, sobre todo en las etapas preco-

Tabla II. Causas de hirsutismo

- Síndrome de ovarios poliquísticos

- Hipertecosis ovárica

- Hirsutismo idiopático o familiar

- Hiperplasia suprarrenal congénita

- Hipotiroidismo

- Síndrome y enfermedad de Cushing

- Medicamentos (hidantoínas, corticoides, esteroides, ciclosporina, diazóxido...) 
ces, el hirsutismo de la hipertricosis. Esta última se caracteriza por la presencia de vello débil y fino pero en zonas habituales, que es más dependiente de factores genéticos y raciales y menos hormonales.

Afecta a niñas en la época puberal y postpuberal como consecuencia de un hiperandrogenismo (tabla II), que es la base fundamental de esta alteración y que puede deberse al aumento de su producción, de la biodisponibilidad por disminución de las proteínas transportadoras (SHRG), al bloqueo de la formación de estrógenos a partir de los andrógenos, a la administración exógena, o a un aumento de la sensibilidad periférica a dichas hormonas. Tanto la glándula suprarrenal como el ovario son los productores de andrógenos en la adolescente. En particular, en el ovario la LH estimula la producción de androstenodiona y testosterona en las células de la teca y posteriormente en las células de la granulosa por acción de la FSH se aromatizan a estrógenos.

No existe correlación entre la expresión clínica y los niveles circulantes de andrógenos. Las causas más frecuentes de hirsutismo quedan reflejadas en la tabla II. En el período prepuberal la causa más frecuente es el hiperandrogenismo suprarrenal y en el período puberal el ovario es responsable de la mayoría de los casos.

\section{Enfoque diagnóstico y pruebas complementarias}

Preguntar a la paciente y/o la familia desde cuándo presenta el vello, la distribución y sus características; presencia de acné o caída del cabello; fecha de la menarquia si ya se produjo, y características de la misma; y posible ingesta de medicación.

En los antecedentes familiares, buscar datos de la misma patología u otras asociadas.

A la exploración valorar la presencia y distribución del vello mediante la escala de Ferriman-Gallway que puntúa de 0 a 4 en nueve áreas corporales y es patológico con una puntuación global superior a 7 (figura 2) ${ }^{12,13}$. Presencia de acné, alopecia, bocio, obesidad con estrías y signos de acantosis nígricans, y en caso extremo en adolescentes explorar las mamas para descartar atrofia mamaria.

Las pruebas complementarias incluyen realizar una determinación basal entre el 3. ${ }^{\circ}$ y $8 .^{\circ}$ día del ciclo menstrual, o tras 2 meses de amenorrea de 17-OH-progesterona, androstenodiona, testosterona libre y total, FSH, LH, estradiol, DHEA-S y SHBG. Además solicitar cortisol, hormonas tiroideas y prolactina. Si la paciente 
Figura 2. Escala de Ferriman-Gallway modificada. Moncada $E^{13}$.

(n)

presenta obesidad, determinar la glucemia, insulina y estudio lipídico.

Para localizar el lugar de producción de andrógenos existen diferentes test dinámicos:

- Test de ACTH (250 mcg intravenoso): determinaciones a los 0 y $60 \mathrm{mi}-$ nutos de 17-OH-progesterona, 17$\mathrm{OH}$-pregnenolona, 11-deoxicortisol, androstenodiona, cortisol y DHEA-S.

- Test Leuprolide (Procrin ${ }^{\circledR} 500$ mcg subcutáneo): determinaciones a las 0 y 3 horas de $\mathrm{LH}, \mathrm{FSH}$, estradiol y a las 24 horas de 17-OH-progesterona ${ }^{14}$.
Valores de 17-OH-progesterona elevados harán sospechar una hiperplasia suprarrenal; una DHEA-S y testosterona muy elevadas, un tumor suprarrenal; la elevación del cortisol y andrógenos, la presencia de una enfermedad de Cushing; valores de LH y FSH normales con una relación $\mathrm{LH} / \mathrm{FSH}>2,5$ sugerirán el diagnóstico de SOP. Si los valores hormonales de FSH y LH son normales pero con andrógenos elevados hablaremos de hipertecosis ovárica, alteración que se debe a la proliferación de las células de la teca y es necesaria la biopsia para el diagnóstico. 
El hirsutismo idiopático suele ser familiar y empezar en la pubertad. Las reglas son normales, también los valores hormonales y se debe al aumento de la sensibilidad de la piel a los andrógenos.

En caso de alteración de las hormonas tiroideas el hirsutismo se produce por disminución de la proteína transportadora de la testosterona, aumentando la testosterona libre.

Las pruebas de imagen tienen valor fundamentalmente para el diagnóstico de patología tumoral.

El tratamiento del hirsutismo precisa una valoración conjunta por el endocrinólogo, dermatólogo y ginecólogo. Depende de la causa en general y de la clínica que predomine. Descartada patología de base, el tratamiento de elección es la administración de un anticonceptivo inicialmente (ver tratamiento del SOP), y un antiandrógeno (flutamida, acetato de ciproterona, espironolactona, etc.) posteriormente, aunque la experiencia con algunos de estos antiandrógenos es escasa. La duración del tratamiento será de al menos 9-12 meses, aunque muchos autores recomiendan un mínimo de 2 años. La pérdida de peso es necesaria en las pacientes con sobrepeso. Además, se debe recurrir a tratamiento estético, que en el caso de las adolescentes mayores sería la fotodepilación-láser que parece ser más eficiente que la electrolisis ${ }^{15,16}$.

\section{Síndrome de ovarios poliquísticos} (SOP)

Es la endocrinopatía más frecuente en mujeres en edad reproductiva (5\%), se caracteriza por hiperandrogenismo (acné, hirsutismo, alopecia, etc.), disfunción menstrual (amenorrea u oligomenorrea con ciclos que pueden ser anovulatorios) y ovarios con morfología poliquística, aunque no siempre. La mitad de los casos no cumple todos los criterios clásicos del síndrome de Stein Leventhal: alteraciones menstruales, hirsutismo, obesidad y ovarios poliquísticos ${ }^{17}$. La importancia de este síndrome se debe no solo a su frecuencia, sino también por ser la causa más frecuente de infertilidad anovulatoria y de hirsutismo ${ }^{18}$.

Las alteraciones hormonales comienzan en la adolescencia e incluyen hiperinsulinismo por insulinorresistencia, sin necesidad de obesidad, y aumento de la frecuencia de los pulsos de LH, dando lugar ambos a una sobreproducción ovárica y adrenal de andrógenos, presumiblemente debido a una disregulación del citocromo P450c ovárico ${ }^{18-20}$. La situación de resistencia insulínica da lugar en el tiempo a una tolerancia alterada a la glucosa, y diabetes mellitus tipo 2. También 
se han descrito alteraciones del perfil lipídico y aumento del riesgo cardiovascular por alteraciones en la fibrinólisis ${ }^{21}$. Por lo tanto, la base fundamental de esta alteración es una situación de hiperandrogenismo, fundamentalmente ovárico.

Parece ser una alteración poligénica que se trasmite de forma dominante $y$ se han valorado diferentes factores, tanto hereditarios como no. La obesidad y la resistencia insulínica, así como factores en la vida embrionaria (exposición a andrógenos, etc.) han sido implicados en la etiopatogenia del SOP'22.

Diferentes alteraciones hormonales en la infancia, como la hiperplasia suprarrenal congénita y la adrenarquia precoz exagerada, darían lugar en el tiempo a esta alteración (tabla III) ${ }^{23}$. La identificación de las niñas con mayor riesgo sería imprescindible para la prevención y tratamiento adecuado, aunque se necesitan más estudios que definan claramente el papel de estos factores en su etiopatogenia.
El diagnóstico en la adolescencia es difícil, porque las irregularidades en la regla, los ciclos anovulatorios y el acné son comunes en la adolescente normal. Todas las adolescentes con los factores previamente descritos deben controlarse por el mayor riesgo.

En el apartado del hirsutismo está reflejado el enfoque diagnóstico, que se basa en la historia clínica, antecedentes familiares muy frecuentes, los antecedentes personales, así como la exploración física y los estudios hormonales.

Cuando la respuesta de la $17-\mathrm{OH}-$ progesterona está elevada en el test de Leuprolide con respuesta normal en el test de ACTH, el hiperandrogenismo es ovárico. Si la respuesta a ambos test está elevada, el hiperandrogenismo es mixto. Para confirmar el origen suprarrenal es preciso realizar un test de supresión con dexametasona $(0,5 \mathrm{mg} / 8$ horas/5 días) determinando $17-\mathrm{OH}$ progesterona, testosterona y DHEA-S. Si estos andrógenos se suprimen en

Tabla III. Secuencia del riesgo del síndrome de ovario poliquístico (SOP) en la infancia (factores de riesgo según la edad)

\begin{tabular}{lll}
\hline Prenatal & Infancia & Adolescencia \\
\hline Peso elevado en la madre & $\begin{array}{l}\text { Pubarquia prematura } \\
\text { Obesidad }\end{array}$ & $\begin{array}{l}\text { Acné } \\
\text { Hirsutismo }\end{array}$ \\
\hline Virilización congénita & Acantosis nigricans & Anovulación \\
\hline Bajo peso al nacer & Síndrome metabólico & Síndrome de ovario poliquístico (SOP) \\
\hline
\end{tabular}


presencia de niveles de cortisol suprimidos ( $\leqslant 1 \mathrm{mcg} / \mathrm{dl}$ ) se confirma el origen suprarrenal. En las pacientes con hiperandrogenismo ovárico puede estar alterado el test de tolerancia a la glucosa con valores de insulina muy elevados (> 100-150 $\mu \mathrm{U} / \mathrm{ml}$ ).

\section{Tratamiento}

Las líneas del tratamiento recomiendan realizar ejercicio físico y alimentación equilibrada para evitar el aumento de peso, o favorecer su disminución, ya que la pérdida de peso permite aumentar la sensibilidad a la insulina, y disminuye los niveles de andrógenos circulantes.

Las guías de consenso aconsejan los anticonceptivos orales como de elección (progestágenos no androgénicos como los que contienen etinilestradiol y drosprenona, como Yasmine ${ }^{\circledast}$, Yasminelle ${ }^{\circledast}$, o etinilestradiol y acetato de ciproterona como Diane ${ }^{\circledast}$ ), añadiendo un antiandrógeno (flutamida, espironolactona, etc.) si a los 6 meses aproximadamente la respuesta no es positiva. Los antiandrógenos solos no están recomendados si no hay una anticoncepción adecuada por el riesgo fetal.

En las pacientes con SOP e hiperinsulinismo, la metformina parece ser una opción terapéutica, pero faltan estudios a largo plazo y no se recomienda en el momento actual.

La eflornitina tópica que inhibe el crecimiento del vello parece reducir el tiempo de remisión, en mujeres adultas ${ }^{24-26}$.

Las técnicas cosméticas se recomiendan igual que en el hirsutismo.

\section{Bibliografía}

1. Moura de Melo MB, Longi CA, Roche MN, Monte $\mathrm{Q}$. Mutation screening in angiotensin II receptors, AGTR1 and AGTR2, and evaluation of AGTR1 polimorphims C573T and A1166C in patients with premature adrenarche. Arq Bras Endocrinol Metab. 2006;50:893-900.

2. Vottero A, Capelletti M, Giliodori S, Vicai I, Ziveri $M$, Neri $M$, Bernasconi $S$, et al. Decreased androgen receptor gene methylation in premature pubarche: a novel pathogenetic mechanism? I Clin Endocrinol Metab. 2006;91:968-72.
3. Roldan MB, White $C$, Witchel $S$. Association of the GAA1013-GAG polimorphism of the insulin-like growth factor-1 receptor (IGF1R) gene with premature pubarche. Fertl Steril. 2007;88:410-7.

4. Lappalainen $S$, Utriainen $P$, Kuuslasmaa $A$, Voutilainen $\mathrm{R}$, Jaaskelainen J. Androgen receptor gene CAG repeat polymorphism and X-chromosome inactivation in children with premature adrenarche. J Clin Endocrinol Metab. 2007;93:1304-9.

5. Ibáñez L, Virdis R, Potau N. Natural history of premature pubarche: an auxological study. J Clin Endocrinol Metab. 1992;74:1317-24. 
6. Ibáñez L, Potau N, Francois I, De Zeguer F. Precocious pubarche, hyperinsulinaemia, and ovarian hyperandrogenism in girls: relation to reduced fetal growth. J Clin Endocrinol Metab. 1998;83: 3558-62.

7. De Zegher F. Prenatal growth restraint followed by catch up of weight; a hyperinsulinemic pathway to polycystic ovary syndrome. Fertil Steril. 2006;86 Suppl 1:s4-s5.

8. Meas T, Chevenne D, Thibaud E, Leger J, Cabrol $S$, Czernichow $P$, et al. Endocrine consequences of premature pubarche in post-pubertal Caucasian girls. Clin Endocrinol (Oxf). 2002;57:101-6.

9. Rosenfield RL. Identifying children at risk for polycystic ovary syndrome. J Clin Endocrinol Metab. 2007;92:787-96.

10. Buggs C, Rosenfield RL. Polycistic ovary syndrome in adolescent. Endocrinol Metab Clin North Am. 2005;34:677-705.

11. Ibáñez L, Potau N, Chacon P, Pascual C, Carrascosa A. Hyperinsulinaemia, dyslipaemia and cardiovascular risk in girls with history of premature pubarche. Diabetología. 1998;41:105763.

12. Ferriman D, Gallwey JD. Clinical assessent of body hair growth in women. J Clin Endocrinol Metab. 1961;21:1440-7.

13. Moncada E. Familial study of hirsutism. J Clin Endocrinol Metab. 1970;31(5):556-64.

14. Ibáñez $L$, Potau $N$, Zampolli $M$, Virdis $R$, Gussinye $M$, Carrascosa $A$, et al. Use of leuprolide acetate response patterns in the early diagnosis of pubertal disorders: comparison with the gonadotropin-releasing hormone test. J Clin Endocrinol Metab. 1994;78:30-5.

15. Swiglo BA, Cosma M, Flynn D, Kurtz D, LaBella ML, Mullan $\mathrm{R}$, et al. Antiandrogens for the treatment of hirsutism: a systematic review and metaanalyses of randomized controlled trials. J Clin Endocrinol Metab. 2007;4:1153-60.
16. Martin KA, Chang RJ, Ibanez L, Lobo RA, Rosenfield R, Shapiro J, et al. Evaluation and treatment of hirsutism in premenopausal women: an endocrine society clinical practice guideline. J Clin Endocrinol Metab. 2008;94:1105-20.

17. Azziz R, Carmina E, Dewailly D, DiamantiKandarakis $\mathrm{E}$, Escobar-Morreale HF, et al. Criteria for defining polycystic ovary syndrome as a predominantly hyperandrogenic syndrome: an androgen excess society guideline. J Clin Endocrinol Metab. 2006; 91:4237-45.

18. Franks S. Polycystic ovary syndrome. N Engl J Med. 1995;333:853-61.

19. Chahabra S, McCanrtney CR, Yoo RY, Eagleson CA, Chang RJ, Marshall JC. Progesterone inhibition of the hypothalamic gonadotropin-releasing hormone pulse generator evidence for varied effects in hyperandrogenemic adolescents girls. J Clin Endocrinol Metab. 2005;90:2810-5.

20. Escobar-Morreale HF, Luque Ramírez M, San Millán JL. The molecular genetic basis of functional hyperandrogenism and polycystic ovary syndrome. Endocr Rev. 2005;26:251-82.

21. Blank SK, Helm KD, McCartney CR, Marshall JC. Polycystic ovary syndrome in adolescence. Ann NY Acad Sci. 2008;1135:76-84.

22. Abbott DH, Barnett DK, Bruns CM, Dumesic DA. Androgen excess fetal programming of female reproduction: a development aetiology for polycystic ovary syndrome? Hum Reprod Update. 2005;11:357-74.

23. Sullivan SD, Moenter SM. Prenatal androgens alter GABAergic drive to gonadotropin releasing hormona neurons: implications for a common fertility disorder. Porc Natl Acad Sci USA. 2004; 101:7129-34.

24. Ibáñez Jaramillo $A M$, Ferrer $A$, de Zegher $F$. Absence of hepototoxicity alter long-term, low dose flutamide in hyperandrogenic girls and young women. Hum Reprod. 2005;20:1833-6. 
25. Cosma $M$, Swiglo BA, Flynn D, Kurtz DM, LaBella ML, Mullan RJ, et al. Insulin sensitizers for the treatment of hirsutim: a systemic review and metaanalyses of randomized con- trolled trials. J Clin Endocrinol Metab. 2008; 93:1135-42.

26. Carmine E. Topical eflornitine: a viewpoint. An J Clin Dermatol. 2001;2:202-4.

\section{ख्ञ⿰氵}

